Z Gerontol Geriat 2019 · 52:654-660 https://doi.org/10.1007/s00391-018-01469-0 Eingegangen: 28. Februar 2018

Überarbeitet: 11. Juli 2018

Angenommen: 17. Oktober 2018

Online publiziert: 30 . Oktober 2018

(c) Der/die Autor(en) 2018

CrossMark

\author{
Jessica Monsees ${ }^{1}$. Wolfgang Hoffmann ${ }^{1,2}$. Jochen René Thyrian' \\ ' Deutsches Zentrum für Neurodegenerative Erkrankungen e.V. (DZNE) Standort Rostock/Greifswald, \\ Greifswald, Deutschland \\ ${ }^{2}$ Abteilung Versorgungsepidemiologie und Community Health, Institut für Community Medicine, \\ Greifswald, Deutschland
}

\title{
Prävalenz von Demenz bei Menschen mit Migrations- hintergrund in Deutschland
}

schen mit Demenz und ihre pflegenden Angehörigen. Beim Umgang mit der Demenz sind nicht nur medizinische und pflegerische Aspekte zu beachten. Genauso wichtig ist ein biografischer, sozialer, kultureller und spiritueller $\mathrm{Zu}$ gang zu den betroffenen Menschen, ihren Familien und ihren pflegenden Angehörigen $[18,22]$. Demenzversorgung muss kultursensibel sein!

Eine Handlungsempfehlung der Allianz besagt deshalb: „Das Versorgungssystem muss daher individuell ausgerichtet sein und passende Unterstützungsangebote [..] bieten [...] für Menschen mit Migrationshintergrund [...] " [8]. In Deutschland lebten im Jahr 2013 16,5 Mio. Menschen mit Migrationshintergrund. Das waren 20,5\% der Gesamtbevölkerung [9]. Bei geschätzten 1,5 Mio. Menschen mit Migrationshintergrund, die älter als 65 Jahre sind, wird die Gruppe der Demenzerkrankten eine relevante Größe in der Versorgung darstellen. Der Wissensstand über diese Gruppe ist jedoch derzeit unzureichend. Migrationshintergrund stellt einen Risikofaktor für die Gesundheit dar [4]. Besondere Gruppen von Migranten nehmen verfügbare Gesundheitsleistungen [16], darunter auch Pflegeleistungen [25], kaum in Anspruch. Da die Demenz in Deutschland unterdiagnostiziert ist [13], ist davon auszugehen, dass dies bei der Gruppe der Migranten eher noch ausgeprägter der Fall ist. Gründe für die geringere Inanspruchnahme werden auf Sprachbarrieren/Analphabetismus [2], kulturelle Unterschiede im Verständ- nis von Krankheit/Gesundheit oder im Umgang damit [3, 17, 18] oder das Fehlen kulturspezifischer Informationen und Angebote [2] zurückgeführt. Ein erheblicher Anteil von Personen mit Migrationshintergrund wird nur schwer vom Gesundheitssystem erreicht, und, wo dies gelingt, kommt es häufig zu Problemen in der Verständigung [2, 21]. Eine differenzierte Schätzung der Anzahl an Demenz erkrankter Menschen mit verschiedenem Migrationshintergrund ist notwendig, um die Größe und Art der Herausforderung auf das Gesundheitssystem abschätzen und ihr durch differenzierte Planung begegnen zu können. Eine Studie aus dem Jahre 2014 schätzte die Zahl der Menschen mit Migrationshintergrund mit einer demenziellen Erkrankung auf 105.543 [11]. Jedoch beruht diese Schätzung nicht auf länderspezifische Prävalenzen. Stattdessen wurden die für Deutschland geltenden Prävalenzzahlen auf die verschiedenen Gruppen angewandt. Zudem wurde für die Analyse nicht nach einzelnen Ethnien unterschieden, sondern generell nach Menschen mit Migrationshintergrund, Ausländern und (Spät-)Aussiedlern.

Das Ziel der vorliegenden Analyse ist eine nach Bundesländern und Prävalenzen in den Heimatländern der verschiedenen Ethnien differenzierte Schätzung der Zahl an Demenz erkrankter Menschen mit Migrationshintergrund in Deutschland. 
Tab. 1 Menschen mit Migrationshintergrund ( $\mathrm{MmM})$ und Demenz in Deutschland

\begin{tabular}{|c|c|c|c|}
\hline & $\begin{array}{l}\mathrm{MmM} \\
n\end{array}$ & $\begin{array}{l}>65 \\
n\end{array}$ & $\begin{array}{l}\text { Mit Demenz } \\
n(\%)\end{array}$ \\
\hline Total & 18.576 .000 & 1.862 .000 & $96.474(5,18)$ \\
\hline 1. Generation & - & 1.814 .000 & - \\
\hline Brandenburg & 162.000 & 15.000 & $649(4,33)$ \\
\hline 1. Generation & - & 14.000 & - \\
\hline Berlin & 991.000 & 79.000 & $3939(4,99)$ \\
\hline 1. Generation & - & 78.000 & - \\
\hline Baden-Württemberg & 3.251 .000 & 357.000 & $18.084(5,07)$ \\
\hline 1. Generation & - & 349.000 & - \\
\hline Bayern & 2.956 .000 & 317.000 & $16.710(5,27)$ \\
\hline 1. Generation & - & 310.000 & - \\
\hline Bremen & 205.000 & 20.000 & $944(4,72)$ \\
\hline 1. Generation & - & 19.000 & - \\
\hline Hessen & 1.870 .000 & 171.000 & $8440(4,94)$ \\
\hline 1. Generation & - & 168.000 & - \\
\hline Hamburg & 538.000 & 46.000 & $2394(5,20)$ \\
\hline 1. Generation & - & 45.000 & - \\
\hline Mecklenburg-Vorpommern & 102.000 & 11.000 & $472(4,29)$ \\
\hline 1. Generation & - & 10.000 & - \\
\hline Niedersachsen & 1.557 .000 & 150.000 & $7194(4,80)$ \\
\hline 1. Generation & - & 147.000 & - \\
\hline Nordrhein-Westfalen & 4.873 .000 & 500.000 & $25.995(5,20)$ \\
\hline 1. Generation & - & 482.000 & - \\
\hline Rheinland-Pfalz & 920.000 & 86.000 & $4057(4,72)$ \\
\hline 1. Generation & - & 83.000 & - \\
\hline Schleswig-Holstein & 414.000 & 42.000 & $1770(4,21)$ \\
\hline 1. Generation & - & 40.000 & - \\
\hline Saarland & 199.000 & 19.000 & $826(4,35)$ \\
\hline 1. Generation & - & 19.000 & - \\
\hline Sachsen & 267.000 & 23.000 & $944(4,10)$ \\
\hline 1. Generation & - & 22.000 & - \\
\hline Sachsen-Anhalt & 140.000 & 15.000 & $649(4,33)$ \\
\hline 1. Generation & - & 15.000 & - \\
\hline Thüringen & 131.000 & 12.000 & $531(4,43)$ \\
\hline 1. Generation & - & 12.000 & - \\
\hline
\end{tabular}

\section{Methode}

Die Analyse basiert auf der Zusammenführung verschiedener, öffentlich verfügbarer Datensätze zur Prävalenz von Demenz und zu Bevölkerungszahlen von Menschen mit Migrationshintergrund.

Die Definition des Statistischen Bundesamtes beschreibt, dass „einen Migrationshintergrund hat, wer durch Geburt nicht die deutsche Staatsangehörigkeit besitzt oder wer mindestens einen Elternteil hat, der durch Geburt nicht die deutsche Staatsangehörigkeit besitzt". sind und eine diagnostizierte Demenz aufweisen, die nach klinischen Kriterien festgestellt wurde [1, 19].

Für die Analysen wurden folgende $\mathrm{Da}$ tensätze verwendet:

1. Dementia in Europe. Yearbook 2013 der Alzheimer Europe. Alzheimer Europe listet hier für verschiedene europäische Länder die Anzahl der Demenzerkrankten in verschiedenen Alterskategorien auf [1].

2. World Population Prospects. Key findings \& advance tables der United Nations (2017) führt für verschiedene Länder die Gesamtpopulation auf [27].

3. World Population Prospects Volume II: Demographic Profiles der United Nations (2017). Die United Nations geben hier an, wie hoch der prozentuale Anteil der über 65-Jährigen an der jeweiligen Population eines Landes ausfällt [28].

4. World Alzheimer Report $2015 \mathrm{der}$ Alzheimer's Disease International. Hier werden Prävalenzzahlen für Personen ab 60 Jahren verschiedener Weltregionen, wie Europa, Asien oder Afrika und deren Subregionen, angegeben [19].

5. Bevölkerung und Erwerbstätigkeit. Bevölkerung mit Migrationshintergrund - Ergebnisse des Mikrozensus 2016 des Statistischen Bundesamtes (2017). Im Mikrozensus wurden im Rahmen einer Zufallsstichprobe 365.700 Haushalte mit 744.000 Personen u. a. $\mathrm{zu}$ verschiedenen demografischen und soziodemografischen Merkmalen befragt. Die hier genutzte Fassung fokussiert dabei auf die Lage der Menschen mit Migrationshintergrund [9].

(Spät-)Aussiedler und Eingebürgerte, aber auch deren Kinder [9]. Soweit möglich wird diese Differenzierung in den Analysen berücksichtigt. Die Daten des Statistischen Bundesamtes werden nach Geschlecht und Bundesland getrennt aufgeführt.

Die Konzeptualisierung von Demenz orientiert sich an den Vorgaben, die Alzheimer's Disease International und Alzheimer Europe für ihre Berechnungen zugrunde gelegt haben. Diese schließen Menschen ein, die 60 bzw. 65 Jahre alt
Um angeben zu können, wie viele Menschen mit einem Migrationshintergrund in Deutschland schätzungsweise eine demenzielle Erkrankung aufweisen, wurden im ersten Schritt Prävalenzen für Demenz der verschiedenen Herkunftsländer ermittelt. Dafür wurden für Bulgarien, Frankreich, Griechenland, Italien, Kroatien, Niederlande, Österreich, Polen, Portugal, Rumänien, Spanien, die Türkei und das Vereinigte Königreich diese Prävalenzen anhand des 
Z Gerontol Geriat 2019·52:654-660 https://doi.org/10.1007/s00391-018-01469-0

(c) Der/die Autor(en) 2018

\section{J. Monsees · W. Hoffmann · J. R. Thyrian}

\section{Prävalenz von Demenz bei Menschen mit Migrationshintergrund in Deutschland}

\section{Zusammenfassung}

Hintergrund. In Deutschland lebten 2013

16,5 Mio. Menschen mit Migrationshintergrund, wovon schätzungsweise $9 \%$ älter als 65 Jahre sind. Sie zeigen häufig schlechtere Gesundheitsoutcomes als Menschen ohne Migrationshintergrund, aber aus verschiedenen Gründen werden Gesundheitsund Pflegeleistungen kaum genutzt. Für diese Population gibt es keine systematischen Angaben zur Anzahl Demenzerkrankter. Dies hat zur Folge, dass das Gesundheitssystem vor einer Herausforderung steht, dessen Dimension kaum einzuschätzen ist.

Ziel. Darstellung der Prävalenz von Demenz bei Menschen mit Migrationshintergrund für Deutschland und die einzelnen Bundesländer.
Methode. Länderspezifische Daten zu Bevölkerung, Demenzerkrankten und altersspezifischen Prävalenzen wurden genutzt, um länderspezifische Prävalenzen für verschiedene Ethnien zu berechnen. Daraus wurde die Anzahl von Menschen mit Migrationshintergrund und Demenz in Deutschland geschätzt, und wie diese sich auf die Bundesländer verteilen.

Ergebnisse. Von 1,86 Mio. Menschen mit Migrationshintergrund in Deutschland, die 65 Jahre oder älter sind, sind schätzungsweise 96.500 Personen (5,2\%) an Demenz erkrankt. Der Großteil entfällt auf Menschen mit polnischem (13.960), italienischem (8920) und türkischem (8840) bzw. europäischem Hintergrund (84.490). Die vermutlich höchsten
Erkrankungszahlen zeigen NordrheinWestfalen (26.000), Baden-Württemberg (18.080) und Bayern (16.710).

Diskussion. Unsere Analysen zeigen die Anzahl an Demenz erkrankter Menschen mit Migrationshintergrund separat für ausgewählte Herkunftsländer als auch die deutschen Bundesländer. Dies bildet eine erste Grundlage für eine länderspezifische Handlungssteuerung und die Planung kultursensibler Angebote zur Versorgungsverbesserung der an Demenz erkrankten Menschen mit Migrationshintergrund.

Schlüsselwörter

Demenz · Migration · Prävalenz $\cdot$ Versorgung · Deutschland

\section{Prevalence of dementia in people with a migration background in Germany}

\section{Abstract}

Background. In Germany the number of people with a migration background was 16.5 million people in 2013 with 9\% being older than 65 years. In this population utilization of health services is low, especially in older people, although they often display worse health outcomes. Little is known about the number of people with dementia and migration background in Germany and their distribution on the state level, resulting in a challenge that is barely assessable for the healthcare system.

Objective. The depiction of the prevalence of dementia in people with a migration background for Germany and the individual federal states.
Method. The number of people with a migration background and dementia living in Germany and the individual federal states was estimated for different ethnic groups using country and state-specific data on the population, dementia frequency and agespecific prevalence.

Results. Out of 1.86 million people with a migration background who are 65 years or older, approximately 96,500 persons (5.2\%) have dementia. The majority are of European $(84,490)$, more specifically Polish $(13,960)$, Italian (8920) and Turkish (8840) heritage. North Rhine-Westphalia $(26,000)$, BadenWuerttemberg $(18,080)$ and Bavaria $(16,710)$ are presumed to show the highest rates of people affected.

Conclusion. In Germany people with a migration background and dementia represent a large target group for health care; however, the numbers and ethnic populations affected differ considerably between states. These analyses can be used for state-specific healthcare planning of culture-specific and culture-sensitive services and care so that specific healthcare improvements can be achieved.

Keywords

Dementia $\cdot$ Migration $\cdot$ Prevalence $\cdot$ Health care -Germany
Dementia in Europe. Yearbook 2013, des World Population Prospects. Key findings \& advance tables und des World Population Prospects Volume II: Demographic Profiles geschätzt, indem der prozentuale Anteil der Demenzerkrankten aus einem Land an dessen Population der über 65-Jährigen bestimmt wurde. Für Bosnien, Kosovo, die Russische Föderation, Serbien, die Ukraine, Marokko, Ägypten, Algerien, Libyen, Tunesien, die Vereinigten Staaten, Irak, Iran, Kasachstan, Syrien, Afghanistan, China, Indien, Pakistan, Vietnam, Australien und Ozeanien wurden die Prävalenzzahlen des „World Alzheimer Report 2015 “ verwendet. Diese länderspezifischen Prävalenzen für Demenz wurden angewandt auf die jeweiligen Gruppen von Menschen mit Migrationshintergrund, die in Deutschland leben und über 65 Jahre alt sind. Die Angaben zu den hier lebenden Menschen mit Migrationshintergrund wurden aus Bevölkerung und Erwerbstätigkeit. Bevölkerung mit Migrationshintergrund - Ergebnisse des Mikrozensus 2016 entnommen. Das Produkt aus der Anzahl von Menschen mit Migrationshintergrund über 65 Jahre einer Ethnie und der entsprechenden Herkunftslandprävalenz stellt die Anzahl derer dar, die in dieser Gruppe eine demenzielle Erkrankung aufweisen.

Die Anzahl an Menschen mit einem Migrationshintergrund und einer Demenz wurde für jedes Herkunftsland bestimmt und daraufhin für die einzelnen Bundesländer und für ganz Deutschland aufaddiert. Anschließend wurde der prozentuale Anteil der Erkrankten an der Population der Menschen mit 
Tab. 2 Ethnien mit den häufigsten Demenzerkrankungen in Deutschland

\begin{tabular}{|c|c|c|c|}
\hline Nationalität & $\begin{array}{l}\text { Prävalenz } \\
\%\end{array}$ & $\begin{array}{l}\text { Anzahl der Menschen } \\
>65 \\
n\end{array}$ & $\begin{array}{l}\text { Anzahl der Menschen mit } \\
\text { Demenz } \\
n\end{array}$ \\
\hline Polen & 7,27 & 192.000 & 13.959 \\
\hline 1. Generation & - & 187.000 & 13.595 \\
\hline Italien & 8,92 & 100.000 & 8920 \\
\hline 1. Generation & - & 98.000 & 8742 \\
\hline Türkei & 4,25 & 208.000 & 8840 \\
\hline 1. Generation & - & 207.000 & 8798 \\
\hline Rumänien & 7,14 & 90.000 & 6426 \\
\hline 1. Generation & - & 89.000 & 6355 \\
\hline Russische Föderation & 4,60 & 137.000 & 6302 \\
\hline 1. Generation & - & 136.000 & 6256 \\
\hline Österreich & 8,24 & 75.000 & 6180 \\
\hline 1. Generation & - & 69.000 & 5686 \\
\hline Kroatien & 7,65 & 73.000 & 5585 \\
\hline 1. Generation & - & 73.000 & 5585 \\
\hline Kasachstan & 4,20 & 96.000 & 4032 \\
\hline 1. Generation & - & 96.000 & 4032 \\
\hline Ukraine & 4,60 & 47.000 & 2162 \\
\hline 1. Generation & - & 46.000 & 2116 \\
\hline Serbien & 4,60 & 34.000 & 1564 \\
\hline 1. Generation & - & 33.000 & 1518 \\
\hline Vereinigte Staaten & 6,40 & 16.000 & 1024 \\
\hline 1. Generation & - & 13.000 & 832 \\
\hline Iran & 4,70 & 16.000 & 752 \\
\hline 1. Generation & - & 16.000 & 752 \\
\hline Marokko & 6,00 & 11.000 & 660 \\
\hline 1. Generation & - & 11.000 & 660 \\
\hline $\begin{array}{l}\text { Ägypten, Algerien, } \\
\text { Libyen, Tunesien }\end{array}$ & 6,00 & 8000 & 480 \\
\hline 1. Generation & - & 8000 & 480 \\
\hline Syrien & 4,70 & 8000 & 376 \\
\hline 1. Generation & - & 8000 & 376 \\
\hline
\end{tabular}

Migrationshintergrund über 65 Jahre errechnet.

Im nächsten Schritt wurde aus diesen Angaben eine Auflistung der 15 Ethnien mit den höchsten geschätzten Erkrankungsfallzahlen in Deutschland zusammengestellt.

Schließlich wurde eine kontinentspezifische Aufschlüsselung der Häufigkeit von Demenzerkrankungen bei Menschen mit Migrationshintergrund erstellt. Hierfür wurden aus dem World Alzheimer Report 2015 die kontinentspezifischen Prävalenzen von Asien, Europa, Amerika, Afrika und Australien übernommen und multipliziert mit der älter als 65 Jahre sind. Von diesen entfällt ein Großteil auf die 1. Generation der hier lebenden Personen mit Migrationshintergrund. In dieser Alterskohorte leiden schätzungsweise 96.480 Menschen an einer Demenzerkrankung. Die meisten Erkrankten finden sich mit ca. 93.800 Erkrankten ebenfalls in der 1. Generation. Die größere Anzahl an Personen mit einer demenziellen Erkrankung findet sich im Westen Deutschlands mit ca. 39.300 Erkrankten (in den Neuen Bundesländern ca. 7200 Erkrankte). In NordrheinWestfalen (ca. 26.000 Erkrankte), BadenWürttemberg (ca. 18.000 Erkrankte) und Bayern (ca. 16.700 Erkrankte) leben die meisten Betroffenen. Die wenigsten in Brandenburg und Sachsen-Anhalt (je ca. 650 Erkrankte), Thüringen (ca. 530 Erkrankte) und Mecklenburg-Vorpommern (ca. 470 Erkrankte) (• Tab. 1). Nach Herkunftsland stammt die größte Gruppe von Menschen mit einer demenziellen Erkrankung aus Polen (ca. 14.000 Erkrankte), gefolgt von Italien (ca. 8920 Erkrankte), Türkei (ca. 8840 Erkrankte), Rumänien (ca. 6430 Erkrankte) und der Russischen Föderation (ca. 6300 Erkrankte) (- Tab. 2). Aus anderen Kontinenten (Asien, Amerika, Afrika und Australien) stammen $12,97 \%$ der an Demenz erkrankten Menschen mit Migrationshintergrund in Deutschland (• Tab. 3).

\section{Diskussion}

Die vorliegenden Analysen zeigen die Anzahl der an Demenz erkrankten Menschen mit Migrationshintergrund auf Basis länderspezifischer Prävalenzzahlen separat nach ausgewählten Herkunftsländern sowie summarisch für alle deutschen Bundesländer. Im Ergebnis leben in Deutschland annähernd 96.500 (ca. 5,2\%) der 1,86 Mio. Menschen mit Migrationshintergrund mit einer demenziellen Erkrankung. Die Schätzung der Gesamtzahl liegt dabei in einem ähnlichen Bereich wie die Schätzung von Drewniok [11]. Es ist jedoch davon auszugehen, dass die tatsächliche Anzahl der Demenzerkrankten höher liegt, da die Demenz unterdiagnostiziert ist $[12,13]$ und daher die Verwendung 
Tab. 3 Häufigkeit von Demenzerkrankungen von MmM in Deutschland, aufgeschlüsselt nach Kontinenten

\begin{tabular}{|l|l|l|l}
\hline Kontinent & $\begin{array}{l}\text { Prävalenz } \\
\%\end{array}$ & $\begin{array}{l}\text { Anzahl der Menschen } \\
>\mathbf{6 5} \\
\boldsymbol{n}\end{array}$ & $\begin{array}{l}\text { Anzahl der Menschen mit } \\
\text { Demenz } \\
\boldsymbol{n}\end{array}$ \\
\hline Europa & $\mathbf{5 , 9 0}$ & $\mathbf{1 . 4 3 2 . 0 0 0}$ & $\mathbf{8 4 . 4 8 8}$ \\
\hline 1. Generation & - & 1.384 .000 & 81.656 \\
\hline EU & 5,90 & 857.000 & 50.563 \\
\hline 1. Generation & - & 826.000 & 48.734 \\
\hline Asien & 4,70 & 191.000 & 8977 \\
\hline 1. Generation & - & 190.000 & 8930 \\
\hline Amerika & 6,40 & 30.000 & 1920 \\
\hline 1. Generation & - & 25.000 & 1600 \\
\hline Nordamerika & 6,40 & 18.000 & 1152 \\
\hline 1. Generation & - & 13.000 & 832 \\
\hline Mittel- und Südamerika & 6,40 & 12.000 & 768 \\
\hline 1. Generation & - & 12.000 & 768 \\
\hline Afrika & 6,00 & 27.000 & 1620 \\
\hline 1. Generation & - & 27.000 & 1620 \\
\hline Australien & 6,70 & - & - \\
\hline 1. Generation & - & - & - \\
\hline
\end{tabular}

der publizierten Prävalenzrate zu einer konservativen Schätzung führt.

Trotz dieser Limitation ist mit diesen Zahlen eine Aussage zu Demenzerkrankten unter den Menschen mit Migrationshintergrund möglich. Bisher gab es nach bestem Wissen der Autoren keine Analyse, die die Prävalenz von Demenz bei Menschen mit Migrationshintergrund in Deutschland ethnien- bzw. kontinentspezifisch dargelegt hat. Die vorliegenden Zahlen können Einrichtungen und Vertretern des Gesundheitsund Versorgungssystems helfen, die Größenordnung der auf sie zukommenden Herausforderung einzuschätzen und bei der Ressourcenplanung, aber auch bei der kultursensiblen Ausrichtung der Angebote helfen. So kann auf Länderebene die Handlungssteuerung verbessert werden, was relevant ist, da die Bundesländer unterschiedliche Verteilungen hinsichtlich der Menschen mit Migrationshintergrund aufweisen (• Tab. 1). Diese Tatsache spiegelt sich entsprechend auch in der Anzahl von Menschen mit einem Migrationshintergrund und einer demenziellen Erkrankung wider, wobei besonders Nordrhein-Westfalen, BadenWürttemberg und Bayern in den kommenden Jahren mit einer deutlich ansteigenden Anzahl an Demenz erkrankter
Menschen mit Migrationshintergrund konfrontiert werden.

Die Allianzfür Menschen mit Demenz hat diesbezüglich in einer Handlungsempfehlung angegeben, dass das Versorgungssystem besser auf Menschen mit Migrationshintergrund eingehen muss [8]. Eine notwendige Voraussetzung für die Umsetzung dieser Empfehlung ist die Kenntnis darüber, wie viele Personen mit welchem Migrationshintergrund betroffen sind. Hier soll die vorliegende Analyse eine Lücke schließen.

Die vorliegenden Hochrechnungen weisen mehrere Einschränkungen auf. Zunächst ist unklar, ob es angemessen ist, für die verschiedenen Ethnien die jeweiligen Prävalenzen der Herkunftsländer zu verwenden. Zudem weisen die Angaben zur Prävalenz z.T. große Unterschiede auf, z. B. sind die Prävalenzen von Italien $(8,92 \%)$, Österreich $(8,24 \%)$ und Kroatien $(7,65 \%)$ wesentlich größer als die Prävalenzen von Kasachstan (4,20\%), der Türkei $(4,25 \%)$ und der Ukraine und Serbien (je 4,6\%) (• Tab. 2). Für diese deutlichen Unterschiede besteht kein berechtigter Grund. Sie könnten aber zum einen durch die angewandte Methodik der Studien erklärt werden. Die Prävalenzraten, die für die Berechnungen herangezogen wurden, beruhen auf Metaanalysen bzw. einem systematischen Review. Die zugrunde liegenden Studien weisen einige Schwächen auf, z. B. in der Qualität oder aufgrund der Tatsache, dass für einige Länder bzw. Regionen mehrStudien bezüglich Prävalenz vorliegen als für andere Länder. Auch Unterschiede im diagnostischen Prozedere der zugrunde liegenden Studien können zu Unterschieden in den resultierenden Prävalenzangaben führen [19]. Zum anderen kann die Erkrankungsrate von Demenz abhängig sein von der ethnischen Zugehörigkeit, was in einem unterschiedlichen Demenzrisiko resultiert. So konnten Studien in den USA aufzeigen, dass Afroamerikaner und Hispanoamerikaner mit 10,5\% bzw. 7,6\% anstelle von 3,4\% ein höheres Risiko für eine Demenz vom Alzheimer-Typ aufweisen. Dabei ist die Zugehörigkeit zur afroamerikanischen bzw. hispanoamerikanischen ethnischen Gruppe direkt und unabhängig mit der Prävalenz von Alzheimer assoziiert [10, 23]. Darüber hinaus ist ein Großteil der Differenz in der Prävalenz von Demenz noch nicht zu erklären.

Durch die Anwendung dieser Prävalenzen auf der Bundeslandebene wird eine Clusterung von Migration nicht berücksichtigt. Demnach könnten durch die Anwendung vorhandene regionale oder lokale Cluster nicht erfasst werden, oder im Gegensatz werden künstliche Cluster gebildet, die nicht vorhanden sind. Nichtsdestotrotz stellt der hier gewählte Ansatz den mit den zurzeit verfügbaren Daten bestmöglichen Ansatz dar.

Eine weitere Limitation betrifft die nicht immer deckungsgleichen Alterskategorien der herangezogenen Datenquellen. So werden die Prävalenzen der Alzheimer's Disease International auf die Gruppe der Personen ab 60 Jahren bezogen, während in der vorliegenden Hochrechnung diese auf die Altersgruppe der über 65-Jährigen angewandt wurde. Dies bedeutet, dass die entsprechenden resultierenden Zahlen von Erkrankten unterschätzt werden. Weiterhin ist anzumerken, dass die Zahlen des Statistischen Bundesamtes auf Hochrechnungen basieren, die gerundet werden, wobei auch widersprüchliche Zahlen resultieren können. Für das Saarland weisen die 
Angaben beispielsweise darauf hin, dass unter den 19.000 Menschen mit Migrationshintergrund, die 65 Jahre oder älter sind, alle über eigene Migrationserfahrungen verfügen, was bedeuten würde, dass es keine Menschen mit Migrationshintergrund in der zweiten Generation gäbe. Genauere Zahlen würden primäre Erhebungen in prospektiven Designs erfordern, die in den betroffenen Gruppen mit erheblichen methodischen Herausforderungen verbunden sind. Jedoch ist es aufgrund vielfältiger Gründe, wie Sprachbarrieren, Angst und Misstrauen, eine Herausforderung, Menschen mit Migrationshintergrund zur Teilnahme an Studien zu bewegen [14, 20]. Zudem besteht die Möglichkeit, dass die hier verwendeten Anzahlen von Personen mit Migrationshintergrund, aber ohne eigene Migrationserfahrung, unterschätzt werden, da diese sich ausschließlich auf Kinder beziehen, die im Haushalt der Eltern leben.

Die hier vorgestellten Hochrechnungen beziehen sich auf das Jahr 2016 und stellen somit nur eine Momentaufnahme dar. Tatsächlich wird der Anteil älterer Menschen mit einem Migrationshintergrund ebenso wie in der deutschstämmigen Bevölkerung ansteigen und damit auch die Prävalenz der demenziellen Erkrankungen. Völlig unklar ist, wie sich die Lage der Menschen der 2. Generation mit einer Demenz darstellen wird. Diese Menschen mit Migrationshintergrund sind in Deutschland geboren und aufgewachsen, deshalb ist anzunehmen, dass sich bei ihnen sowohl Versorgungsbedarfe als auch die Inanspruchnahme von den Personen der 1. Generation unterscheiden. Dies wird in besonderem Maße auf verschiedene Kulturen zutreffen, wobei auch der Erfolg der Integration eine wichtige Rolle spielen wird. Obwohl dies schwer quantitativ belegt werden kann, gehen wir davon aus, dass die Verfügbarkeit von kulturspezifischen Einrichtungen und Angeboten in Deutschland bei Weitem noch nicht ausreicht und in den kommenden Jahren verstärkt aufund ausgebaut werden muss. Gleichzeitig müssen die Bemühungen zur Erfassung von Primärdaten sowohl zur Morbidität als auch zur Versorgungssituation von Menschen mit Migrationshinter- grund fortgesetzt und, wo erforderlich, weiter intensiviert werden $[15,20,24]$.

\section{Fazit für die Praxis}

- Die Gruppe der Menschen mit Demenz und Migrationshintergrund stellt allein von ihrer Anzahl her eine relevante Größe in der Versorgungslandschaft dar. Es besteht Handlungsbedarf bei der Erforschung und kultursensiblen Versorgung der Betroffenen.

- Die Bundesländer sind verschieden stark betroffen. Zum einen, was die Anzahl der Menschen mit Demenz und Migrationshintergrund angeht. Zum anderen, was den Migrationshintergrund angeht. Strategien zum Umgang und zur besseren Versorgung müssen kultursensibel und regional angepasst entwickelt und umgesetzt werden.

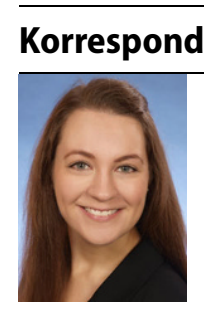

Jessica Monsees
Deutsches Zentrum
für Neurodegenerative
Erkrankungen e.V. (DZNE)
Standort Rostock/Greifswald
Ellernholzstraße 1-2,
17489 Greifswald,
Deutschland
jessica.monsees@dzne.de

\section{Einhaltung ethischer Richtlinien}

Interessenkonflikt. J. Monsees, W. Hoffmann und J.R. Thyrian geben an, dass kein Interessenkonflikt besteht.

Dieser Beitrag beinhaltet keine Studien an Menschen oder Tieren.

Open Access. Dieser Artikel wird unter der Creative Commons Namensnennung 4.0 International Lizenz (http://creativecommons.org/licenses/by/4.0/deed. de) veröffentlicht, welche die Nutzung, Vervielfältigung, Bearbeitung, Verbreitung und Wiedergabe in jeglichem Medium und Format erlaubt, sofern Sie den/die ursprünglichen Autor(en) und die Quelle ordnungsgemäßnennen, einen Link zur Creative Commons Lizenz beifügen und angeben, ob Änderungen vorgenommen wurden.

\section{Literatur}

1. Alzheimer Europe (2013) Dementia in Europe. Yearbook 2013. Alzheimer Europe, Luxemburg

2. Bermejo I, Hölzel LP, Kriston L et al (2012) Subjektiv erlebte Barrieren von Personen mit Migrationshintergrund bei der Inanspruchnahme von Gesundheitsmaßnahmen. Bundesgesundheitsblatt Gesundheitsforschung Gesundheitsschutz 55:944-953

3. Blümel S (2015) Gesundheitliche Aufklärung für Menschen mit Migrationshintergrund. Bundesgesundheitsblatt Gesundheitsforschung Gesundheitsschutz 58:593-600

4. Brzoska P, Voigtländer S, Spallek J et al (2010) Utilization and effectiveness of medical rehabilitation in foreign nationals residing in Germany. Eur J Epidemiol 25:651-660

5. Deutsche Alzheimer Gesellschaft e.V. (2018) Informationsblatt 1: Die Häufigkeit von Demenzerkrankungen. https://www.deutsche-alzheimer. de/fileadmin/alz/pdf/factsheets/infoblatt1_ haeufigkeit_demenzerkrankungen_dalzg.pdf. Zugegriffen: 9. Jan. 2018

6. Bundesministerium für Familie, Senioren, Frauen und Jugend (o.J.) Die Allianz. https://www.allianzfuer-demenz.de/informationen/die-allianz.html. Zugegriffen:09. Januar 2018

7. Bundesministerium für Familie, Senioren, Frauen und Jugend (o.J.) Gesellschaftliche Verantwortung. https://www.allianz-fuerdemenz.de/handlungsfelder/gesellschaftlicheverantwortung.html.Zugegriffen:09. Januar 2018

8. Bundesministerium für Familie, Senioren, Frauen und Jugend (o.J.) Gestaltung des Unterstützungsund Versorgungssystems. https://www.allianzfuer-demenz.de/handlungsfelder/gestaltungdes-unterstuetzungs-und-versorgungssystems. html. Zugegriffen:09. Januar 2018

9. Statistisches Bundesamt (2017) Bevölkerung und Erwerbstätigkeit. Bevölkerung mit Migrationshintergrund - Ergebnisse des Mikrozensus 2016. https://www.destatis.de/DE/Publikationen/ Thematisch/Bevoelkerung/MigrationIntegration/ Migrationshintergrund2010220167004 blob=publicationFile. Zugegriffen: 09. Januar 2018

10. Demirovic J, Prineas R, Loewenstein D et al (2003) Prevalence of dementia in three ethnic groups: the South Florida Program on Aging and Health. Ann Epidemiol 13:472-478

11. Drewniok A (2014) Migration, Pflegebedürftigkeit und Demenz - Ein Versuch einer Standortbestimmung. Pflegewissenschaft 14:452-464

12. Edwards R, Voss S, Iliffe S (2014) Education about dementia in primary care: is person-centredness the key? Dementia 13:111-119

13. Eichler T, Thyrian JR, Hertel J et al (2015) Rates of formal diagnosis of dementia in primary care: the effect of screening. Alzheimers Dement (Amst) 1:87-93

14. Ibrahim S, Sidani S (2014) Strategies to recruit minority persons: a systematic review. J Immigr Minor Health 16:882-888

15. Kristiansen M, Razum O, Tezcan-Güntekin $\mathrm{H}$ et al (2016) Aging and health among migrants in a European perspective. Public Health Rev. https:// doi.org/10.1186/s40985-016-0036-1

16. Lindert J, Priebe S, Penka S et al (2008) Versorgung psychisch kranker Patienten mit Migrationshintergrund. Psychother Psychosom Med Psychol 58:123-129

17. Maier I, Kriston L, Härter M et al (2015) Psychometrische Überprüfung eines Fragebogens 
zur Erfassung der Barrieren der Inanspruchnahme von Gesundheitsleistungen durch Personen mit Migrationshintergrund. Gesundheitswesen 77:749-756

18. Mogar M, von Kutzleben M (2015) Demenz in Familien mit türkischem Migrationshintergrund. ZGerontol Geriatr 48:465-472

19. Prince M, Wimo A, Guerchet M et al (2015) World Alzheimer report 2015. The global impact of dementia. An analysis of prevalence, incidence, cost and trends. Alzheimer's Disease International (ADI), London

20. Razum O, Wenner J (2016) Social and health epidemiology of immigrants in Germany: past, present and future. Public Health Rev. https://doi. org/10.1186/s40985-016-0019-2

21. Spallek J, Razum O (2007) Gesundheit von Migranten:Defizite im Bereich der Prävention.Med Klin Intensivmed Notfmed 102:451-456

22. Spilker RS (2017) Dementia-care services for migrants and ethnic minorities. In: Sagbakken M Kumar B (Hrsg) Dementia, ethnic minorities and migrants. A review of the literature. Norwegian Centre for Migration and Minority Health, Oslo, S 79-97

23. Tang M-X, Stern Y, Marder Ket al (1998) The APOE$\varepsilon 4$ allele and the risk of Alzheimer disease among African Americans, whites, and hispanics. JAMA 279:751-755

24. Tezcan-Güntekin H, Oliver R (2015) Pflege von Menschen mit Migrationshintergrund. Spezifische Bedürfnisse erkennen. Dtsch Arztebl 39:A1564-A1565

25. Thum M, Delkic E, Kemnitz A et al (2015) Auswirkungen des demographischen Wandels im Einwanderungsland Deutschland. FriedrichEbert-Stiftung, Bonn

26. Thyrian JR (2017) Menschen mit Demenz in der primärärztlichen Versorgung. Z Gerontol Geriatr 50:32-38

27. United Nations (2017) World population prospects. The 2017 revision. Key findings \& advance tables. United Nations, New York

28. United Nations (2017) Demographic profiles. World population prospects. The 2017 revision, Bd. II. United Nations, New York

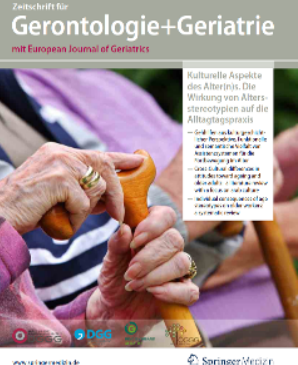

\section{Kulturelle Aspekte des Alter(n)s - die Wirkung von Altersstereotypien auf die Alltagspraxis}

Neues Online-Supplement der Zeitschrift für Gerontologie und Geriatrie H. Fangerau, A. von Hülsen-Esch, D. Schäfer (Hrsg.)

Eine bestimmte Haltung dem Alter und alten Menschen gegenüber fließt oftmals als nicht bewusste Grundannahme in das Regelwerk des gesamtgesellschaftlichen Gefüges ein. Dieser Umgang mit dem Alter ist wiederum geprägt von der Kultur- Kultur aber ist ein schillernder Begriff.

Alter erhält eine Doppelgesichtigkeit. Neben biologisch ermittelbaren Alterungsprozessen, ist die Frage der jeweiligen Interpretation eines Zustands im Sinne einer Einordnung in Symbolund Deutungswelten einer Gesellschaft bedeutsam.

Die Untersuchung kultureller Aspekte des Alters zielt dabei auf den von Menschen erzeugten Gesamtkomplex aus Vorstellungen, Denkformen, Empfindungsweisen, Werten und Bedeutungen, der sich in Symbolsystemen materialisiert. Einer solchen Begriffsbestimmung zufolge sind nicht nur materiale (z.B. künstlerische) Ausdrucksformen zum Bereich der Kultur zu zählen, sondern auch die sozialen Institutionen und mentalen Dispositionen, die die Hervorbringung solcher Artefakte überhaupt erst ermöglichen.

Es geht darum, das Alter(n) selbst als einen kulturellen Prozess zu begreifen, auch, um starre Altersstereotypien, die beispielsweise sprachlich intergenerationell weitergegeben werden, bewusst zu machen und aufzubrechen In diesem Sonderheft werden drei Blickweisen auf kulturelle Aspekte des Alters in diesem Sinne zusammengeführt und dabei interdisziplinäre Spezifika des Blicks auf die Kultur illustriert:

-Gehhilfen aus kulturgeschichtlicher Perspektive. Funktionelle und semantische Vielfalt von Assistenzsystemen für die Fortbewegung im Alter (D. Schäfer, A. von Hülsen-Esch, H. Fangerau)

-Cross-Cultural Differences in Attitudes toward Ageing and Older Adults: A Literature Review with a Focus on Arab Culture (C.N.R. Ibrahim, U.J. Bayen)

-Individual consequences of age stereotypes on older workers: A systematic review (J. Weber, P. Angerer, A. Müller)

Abonnenten der Zeitschrift haben kostenfreien Zugriff auf die Inhalte des Supplements und finden dieses im Online-Archiv der Zeitschrift (Jahrgang 2019) unter: www.springermedizin.de/zeitschrift-fuer-gerontologie-und-geriatrie. 